\title{
Should doctors inform terminally ill patients? The opinions of nationals and doctors in the United Arab Emirates
}

\author{
Ann Harrison, ^ Ahmed M H Al-Saadi, Ali S O Al-Kaabi, Mohammed R S Al-Kaabi, \\ Saif S M Al-Bedwawi, Saif O M Al-Kaabi and Salem B S Al-Neaimi ${ }^{\star} S t$ George's University School of \\ Medicine, Grenada and Faculty of Medicine and Health Sciences, UAE University
}

\section{Abstract}

Objectives - To study the opinions of nationals (Emiratis) and doctors practising in the United Arab Emirates (UAE) with regard to informing terminally ill patients.

Design - Structured questionnaires administered during Fanuary 1995.

Setting - The UAE, a federation of small, rich, developing Arabian Gulf states.

Participants - Convenience samples of 100 Emiratis (minimum age 15 years) and of 50 doctors practising in government hospitals and clinics.

Results - Doctors emerged as consistently less in favour of informing than the Emiratis were, whether the patient was described as almost certain to die during the next six months or as having a 50\% chance of surviving, and even when it was specified that the patient was requesting information. In the latter situation, a third of doctors maintained that the patient should not be told. Increasing survival odds reduced the number of doctors selecting to inform; but it had no significant impact on Emiratis' choices. When Emiratis were asked whether they would personally want to be informed if they had only a short time to live, less than half responded in the way they had done to the in principle question.

Conclusions - The doctors' responses are of concern because of the lack of reference to ethical principles or dilemmas, the disregard of patients' wishes and dependency on survival odds. The heterogeneity of Emiratis' responses calls into question the usefulness of invoking norms to explain inter-society differences. In the current study, people's in principle choices did not provide a useful guide to how they said they would personally wish to be treated.

\section{Introduction}

TRUTH-TELLING BY PHYSICIANS

In the United States, truth-telling by doctors "has

\section{Key words}

Truth-telling; cross-cultural. tended to become a moral absolute"1 justified in terms of the ethical principle of autonomy which requires doctors to respect the right and ability of patients to make decisions. The automatic adoption of any approach, however, is incompatible with optimal ethical management. What is needed from doctors is a sage consideration of each patient's situation and needs, an appropriate prioritising of ethical principles and the selection of effective methods for achieving these. If, for personal, religious or cultural reasons, a patient does not wish to be told, truthtelling is contraindicated both in terms of autonomy and beneficence.

Frequency of full disclosure varies widely between doctors, institutions and countries. ${ }^{234}$ The trend seen in many countries over recent decades has been towards providing patients with more information. ${ }^{125}$ How far this is a response to legal and social developments, and how far it reflects increased empathy and compliance with patients' wishes, is not clear.

\section{CULTURAL FACTORS AND TRUTH-TELLING}

An issue which has only recently been raised is whether cultural differences require different behaviour from physicians. ${ }^{1}$ In a seminal letter, Surbone contrasted truth-telling by oncologists in America and Italy and used cultural differences to explain disparities in behaviour and professional codes. ${ }^{6}$

The term cultural differences subsumes many factors. Among the non-material aspects of a culture are its values, norms, customs, expectations, language and history. Material aspects include what medical services are available and how these are organised. Both are likely to have an impact on what expectations, needs and behaviours are typical of patients and doctors in a given society and different subcultures within the same society.

Some cultures hold that most people are capable of making properly informed decisions about medical issues, others believe that an adequate understanding of medical data is beyond most people. ${ }^{6}$ It follows that the latter will expect doctors 
to make decisions on behalf of their patients. While paternalism has acquired something of a pejorative overtone in the West, it can be argued that if this is what a patient needs or wants, then this is the role the doctor should play. A paternalistic approach should never be adopted, however, simply because it is easier than educating patients to assume greater control or because doctors are overlooking the option of full disclosure. ${ }^{5}$

In North America, it is usual for competent adult patients to control what information family members are given. For Italians, however, it has been argued that autonomy connotes isolation from the family and that informing just the patient may jeopardise family support. ${ }^{6}$ In societies where important personal decisions are left to senior family members, it may be inappropriate to inform just the patient; in clinical settings where family members are continuously present, it may not even be practical. Another consideration is what medical services a patient has access to. For patients who cannot afford a potentially useful therapy and for patients who would not be accepted for treatment, the doctor must consider whether they will derive greater benefit or greater suffering from being told about it.

Doctors cannot provide empathic care unless they are cognisant of their patient's idiosyncratic needs and mindful of relevant cultural issues. For this reason, it is important to substantiate putative cultural differences. Do Italians, as claimed, ${ }^{6}$ find it more difficult than Americans openly to confront sickness and death? Are Mediterranean people so sentimental that they find it difficult to accept a cruel truth? ${ }^{5}$ Do Greeks really consider it irrational for a patient to refuse treatment ${ }^{5}$

\section{CONTRASTING PATIENTS' WISHES AND DOCTORS'}

BEHAVIOUR

The current study sought to compare the opinions of Emirati adults and doctors practising in the UAE about whether or not doctors should inform terminally ill patients. Past studies are said to have focused too often on cancer patients - where disclosure rates are unusually low. ${ }^{7}$ For this reason, the current study did not specify the nature of the illness. Based on an earlier Greek study, ${ }^{5}$ respondents were asked to consider two patients: one almost certain to die within the next six months and the other with a $50 \%$ chance of dying during the same period. The response options available were: "Yes, the doctor should inform the patient", "No, the doctor should not" and "It depends". In the Greek study, ${ }^{5}$ the odds of the patient dying and the time period considered covaried, making it impossible to differentiate what impact each was having. For the current study, the period was fixed at six months. The 500 Greek lay people who participated displayed no consensus. ${ }^{5}$ Males and females did not differ significantly in their views. More educated and younger respondents, however, emerged as more likely to choose "It depends" and less less likely to state that the doct产 should not inform. It is also noteworthy that when called upon to explain their choices, most cited prat tical considerations. Ethical concepts and patients' rights were almost never alluded to.

Gastroenterologists from 27 European countrifs were asked what information they would provide fo $_{0}$ a patient with colonic cancer. ${ }^{3}$ The proportion of doctors in a country who stated that they woutd provide full information varied geographicalts: movements south and east were associated with lès disclosure. The vast majority of Greek doctors who took part stated that they would not volunteer the diagnosis to patients who did not ask. Just over ha्plf said they would give the diagnosis if specificaly requested to by the patient. In the case presentẹd, subsequent resection of the colon revealed metasttases in both liver lobes. Asked how they woutt respond if the patient asked about the operation, only one Greek doctor said that he/she would tell the patient that metastases had been found. None co sidered that the patient should be told the condition was incurable.

The use of different cases when exploring the opinions of Greek lay people ${ }^{5}$ and Greek doctors ${ }^{3}$ precludes any proper comparison of their vieus. These studies ${ }^{35}$ do, however, suggest that the thipd of Greek laypeople who responded unequivocadily that doctors should inform terminally ill patierigs would, if they were suffering from a terminal gastrointestinal condition, be unlikely to be managed by specialist who endorsed this view. In the current study, Emiratis and doctors practising in the USE were presented with identical cases, so that their views could be contrasted.

The UAE is a multicultural society, with a sutbstantial proportion of expatriates. In order to achie a reasonable level of cultural homogeneity, the sample of lay people was restricted to Emiratis. Ethical clearance for the study was provided by the Research Ethics Committee of the Faculty Medicine and Heath Sciences, United Arab Emirates University.

\section{Subjects and methods}

Convenience samples of (a) 100 Emiratis and (b) $\$ 0$ doctors practising in government hospitals aBd clinics in the UAE were interviewed. The only selef tion criteria for Emiratis was that an individual should: be at least 15 years old; be willing to providte written informed consent; not be suffering frompa terminal illness or have a close relative with $\mathrm{\rho}_{\mathrm{a}}$ terminal illness, and not have had anyone close $\overrightarrow{\&}$ him/her die from what had been identified beforehand as a terminal illness during the preceding I2 months. Meeting places catering to a broad cros section of the national population (for exampte, coffee shops, sport and social clubs) were used contacting potential participants. Fewer than $10 \%$ of 


(1)

Table 1: Sample profiles

\begin{tabular}{|c|c|c|c|c|c|}
\hline \multicolumn{3}{|l|}{ Emiratis } & \multicolumn{3}{|l|}{ Doctors } \\
\hline Variable & Categories & $N(\%)$ & Variable & Categories & $N(\%)$ \\
\hline \multirow[t]{2}{*}{ Sex } & \multirow{7}{*}{$\begin{array}{l}\text { Males } \\
\text { Females } \\
15-25 \text { yrs } \\
26-35 \text { yrs } \\
36-45 \text { yrs } \\
46-55 \text { yrs } \\
\text { over } 55 \text { yrs }\end{array}$} & \multirow{7}{*}{$\begin{array}{c}47(47 \%) \\
53(53 \%) \\
50(50 \%) \\
28(28 \%) \\
10(10 \%) \\
6(6 \%) \\
6(6 \%)\end{array}$} & \multirow{7}{*}{$\begin{array}{l}\text { Sex } \\
\text { Field of practice }\end{array}$} & Males & \multirow{11}{*}{$\begin{array}{r}44(88 \%) \\
6(12 \%) \\
21(42 \%) \\
11(22 \%) \\
9(18 \%) \\
3(6 \%) \\
2(4 \%) \\
2(4 \%) \\
2(4 \%) \\
3(6 \%) \\
16(32 \%) \\
20(40 \%) \\
11(22 \%)\end{array}$} \\
\hline & & & & Females & \\
\hline Age & & & & Medicine & \\
\hline & & & & $\begin{array}{l}\text { Surgery } \\
\text { General practice }\end{array}$ & \\
\hline & & & & Orthopaedics & \\
\hline & & & & Oncology & \\
\hline & & & & $\begin{array}{l}\text { Paediatrics } \\
\text { Other }\end{array}$ & \\
\hline \multirow{4}{*}{ Level of education } & Uneducated & $13(13 \%)$ & \multirow{4}{*}{$\begin{array}{l}\text { Years of practice } \\
\text { in the UAE }\end{array}$} & $<1 \mathrm{yr}$ & \\
\hline & Primary & $8(8 \%)$ & & $1-5$ yrs & \\
\hline & Middle & $14(14 \%)$ & & $6-15 \mathrm{yrs}$ & \\
\hline & $\begin{array}{l}\text { Secondary } \\
\text { Higher education }\end{array}$ & $\begin{array}{l}37(37 \%) \\
28(28 \%)\end{array}$ & & 16 plus yrs & \\
\hline
\end{tabular}

the Emiratis interviewed were currently receiving medical care. Doctors working in government hospitals and clinics were approached, and those willing to provide informed consent were interviewed. Ten of the doctors interviewed had trained in Europe, the others were graduates of medical schools in the Middle East, India, Pakistan or Africa. Fourteen of the Emiratis approached, and eight of the doctors, chose not to participate. Profiles of the two samples are provided in table 1 .

Subjects were interviewed using structured questionnaires; interviewers were bilingual (Arabic/ English) second-year medical students. Emiratis were interviewed in Arabic, and the doctors in English. All respondents were asked for their opinions concerning two cases (table 2). The options provided were "Yes, the doctor should inform the patient", "No, the doctor should not" and "It depends". Respondents were then asked what should happen if the patient specifically asked the doctor for information. Subjects were asked to outline what, if any, circumstances make it more and less appropriate for the doctor to inform. Doctors were asked how often they had dealt with cases like the ones described during the past year; and Emiratis were asked to describe any comparable situations they had experienced. Doctors were asked whether patients in the UAE resembled or were different from patients in the West in terms of wishing to be informed when their prognosis was poor. Emiratis were asked how much of what they were told by doctors in the UAE they believed; and, lastly, whether they would want a doctor to tell them if they had only a short time to live.

\section{Results}

COMPARING EMIRATIS' AND DOCTORS' OPINIONS

Doctors and Emiratis differed significantly in their reactions to the cases presented (table 2): fewer doctors felt that patients should be informed, and more believed that the exact circumstances ought to be considered. The same pattern was evident even when it was specified that the patient was requesting information.

\section{WHEN THE PATIENT ASKS TO BE TOLD}

Specifying that the patient was requesting information produced a shift from the "It depends" to the "Yes" option (table 2). This change was significant for both Case 1 (Emiratis: chi $^{2}=35.17$, df $=2$, $\mathrm{p}<0.0000001$; Doctors: chi $^{2}=18.32$, df $=2$, $\mathrm{p}<0.0005$ ) and Case 2 (Emiratis: $\mathrm{chi}^{2}=25.19, \mathrm{df}=2$, $\mathrm{p}<0.000005$; Doctors: $\mathrm{chi}^{2}=17.15$, $\mathrm{df}=2$, $\mathrm{p}<0.0005)$.

Table 2: Emiratis' and doctors' reactions to Case 1 and Case 2

\begin{tabular}{|c|c|c|c|c|c|c|c|}
\hline \multirow[b]{2}{*}{ Options } & \multicolumn{2}{|c|}{ "Yes" } & \multicolumn{2}{|c|}{ "No" } & \multicolumn{2}{|c|}{ "It depends" } & \multirow[b]{2}{*}{ Comparing Emiratis and doctor } \\
\hline & $N$ & $\%$ & $N$ & $\%$ & $N$ & $\%$ & \\
\hline
\end{tabular}

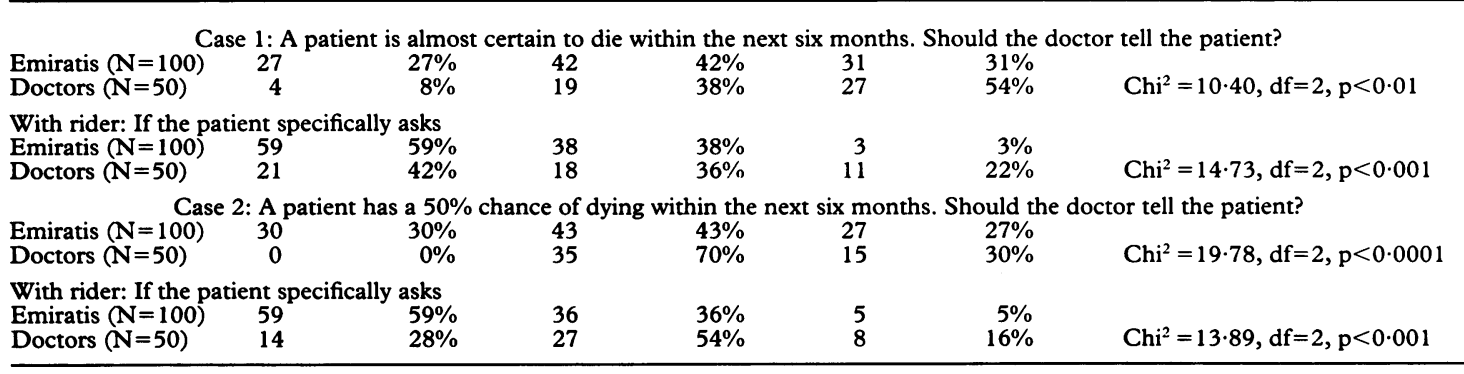


Table 3: Comparing the groups' responses to Cases 1 and 2

\begin{tabular}{|c|c|c|c|c|c|c|c|}
\hline \multirow[b]{2}{*}{ Options } & \multicolumn{2}{|c|}{ "Yes" } & \multicolumn{2}{|c|}{ "No" } & \multicolumn{2}{|c|}{ "It depends" } & \multirow[b]{2}{*}{ Comparing a group's responses } \\
\hline & $N$ & $\%$ & $N$ & $\%$ & $N$ & $\%$ & \\
\hline $\begin{array}{l}\text { Emiratis }(\mathrm{N}=100) \\
\text { Case } 1 \\
\text { Case } 2 \\
\text { Case } 1 \text { with rider } \\
\text { Case } 2 \text { with rider }\end{array}$ & $\begin{array}{l}27 \\
30 \\
59 \\
59\end{array}$ & $\begin{array}{l}27 \% \\
30 \% \\
59 \% \\
59 \%\end{array}$ & $\begin{array}{l}42 \\
43 \\
38 \\
36\end{array}$ & $\begin{array}{l}42 \% \\
43 \% \\
38 \% \\
36 \%\end{array}$ & $\begin{array}{r}31 \\
27 \\
3 \\
5\end{array}$ & $\begin{array}{r}31 \% \\
27 \% \\
3 \% \\
5 \%\end{array}$ & $\begin{array}{l}\mathrm{Chi}^{2}=0.45, \mathrm{df}=2, \mathrm{p}>0.05 \\
\mathrm{Chi}^{2}=0.55 \mathrm{df}=2, \mathrm{p}>0.05\end{array}$ \\
\hline $\begin{array}{l}\text { Doctors }(\mathrm{N}=50) \\
\text { Case } 1 \\
\text { Case } 2\end{array}$ & $\begin{array}{l}4 \\
0\end{array}$ & $\begin{array}{l}8 \% \\
0 \%\end{array}$ & $\begin{array}{l}19 \\
35\end{array}$ & $\begin{array}{l}38 \% \\
70 \%\end{array}$ & $\begin{array}{l}27 \\
15\end{array}$ & $\begin{array}{l}54 \% \\
30 \%\end{array}$ & $\mathrm{Chi}^{2}=12 \cdot 17, \mathrm{df}=2, \mathrm{p}<0.005$ \\
\hline $\begin{array}{l}\text { Case } 1 \text { with rider } \\
\text { Case } 2 \text { with rider }\end{array}$ & $\begin{array}{l}21 \\
14\end{array}$ & $\begin{array}{l}42 \% \\
28 \%\end{array}$ & $\begin{array}{l}18 \\
27\end{array}$ & $\begin{array}{l}36 \% \\
54 \%\end{array}$ & $\begin{array}{r}11 \\
8\end{array}$ & $\begin{array}{l}22 \% \\
16 \%\end{array}$ & $\mathrm{Chi}^{2}=3.66, \mathrm{df}=2, \mathrm{p}>0.05$ \\
\hline
\end{tabular}

THE PROBABILITY OF DYING

Changing survival odds (table 3 ) did not significantly affect Emiratis' judgments. It did, however, have an impact on doctors: when death was less likely, more doctors stated that the patient should not be informed. The same trend was seen when it was stated that the patient was asking for information.

CONTRASTING PEOPLES' PERSONAL WISHES AND IN PRINCIPLE STATEMENTS

At the end of the interview session, Emiratis were asked whether or not they would want the doctor to tell them if they had only a short time to live. Their choices (table 4) differed significantly from their responses to Case $1 \quad\left(\mathrm{chi}^{2}=31.33, \mathrm{df}=2\right.$, $\mathrm{p}<0.0000005)$. Those who stated unequivocally that they would want to be told if they only had a short time to live were divided in their opinions about how the doctor should respond to Case 1 . Those who did not wish to be informed were more likely to make the same choice for Case 1. Those who selected "It depends" for themselves made the same choice for Case 1 .

\section{INFORMING THE FAMILY BUT NOT THE PATIENT}

Asked whether the doctor would ever be justified in telling the family but not the patient: $71 \%$ of Emiratis and $88 \%$ of doctors said "Yes" for Case 1, and $62 \%$ of Emiratis and $80 \%$ of doctors said "Yes" for Case 2. Emiratis mentioned a number of reasons. The most frequent (mentioned by a third) was to

Table 4: Comparing Emiratis' personal and in principle choices

\begin{tabular}{|c|c|c|c|c|c|c|}
\hline \multirow[b]{2}{*}{ Options } & \multicolumn{2}{|c|}{ "Yes" } & \multicolumn{2}{|c|}{ “No" } & \multicolumn{2}{|c|}{ "It depends" } \\
\hline & $N$ & $\%$ & $N$ & $\%$ & $N$ & $\%$ \\
\hline
\end{tabular}

If you had only a short

Personal choice

time to live, would you

want the doctor to tell

$\begin{array}{lllllll}\text { you? }(\mathrm{N}=96) & 55 & 57 \% & 37 & 39 \% & 4 & 4 \%\end{array}$

Reaction to Case 1: A patient is almost certain to die within the "Yes" next six months. Should the doctor tell the patient?

\begin{tabular}{lrrrrrc} 
"Yes" & 18 & $33 \%$ & 7 & $19 \%$ & 0 & $0 \%$ \\
"No" & 18 & $33 \%$ & 23 & $62 \%$ & 0 & $0 \%$ \\
"It depends" & 19 & $34 \%$ & 7 & $19 \%$ & 4 & $100 \%$ \\
\hline Total & 55 & $100 \%$ & 37 & $100 \%$ & 4 & $100 \%$ \\
\hline
\end{tabular}

encourage the family to provide greater care. Otheî justifications were if the patient was psychologicallio unstable, too young or too old, or if the conditiob was contagious or inheritable. About $7 \%$ felt that families should be left to tell the patient in their way? Twenty-two per cent of doctors claimed that the $\vec{y}$ always informed the family, the remainder justifie informing the family in terms of safeguarding family support, the age and psychological status of the patient and enabling the family to establish the pos. sibility of treatment elsewhere.

\section{HOW RESPONDENTS EXPLAINED THEIR CHOICES}

Emiratis were asked to explain why they believed patient should or should not be informed, and to outline any conditions which they felt should encourage or discourage doctors from informings None mentioned that it was the right of the patient to know or the ethical responsibility of the doctor inform. Practical issues were mentioned, for example, settling finances, making a will, securing the family's future, avoiding additional risks, giving the patient an opportunity to travel abroad for treat ment, and justifying isolation if the patient had 8 contagious condition. Religious reasons were offere to justify both telling (providing the person with as opportunity to pray and prepare) and not telling ( $s Q$ that the person does not lose faith). Some people fele that only psychologically stable patients should be informed. None of these points, however, was mentioned by more than a third of respondents.

When doctors were asked whether there were conditions which would make them feel more como fortable or less comfortable about informing, doctors mentioned a range of factors including the age, se and education of the patient, the patient's family and financial status, psychological stability, strength of religious belief, access to treatment abroad and the doctor's confidence in the diagnosis. Only one doctor mentioned an ethical issue, stating that $\mathrm{h} \mathbb{\mathrm { B }}$ would feel less comfortable about informing the patient if he could not ensure confidentiality.

Male and female Emiratis did not differ in thei responses to Case $1\left(\mathrm{chi}^{2}=1.24, \mathrm{df}=2, \mathrm{p}>0.05\right)$ or 
Table 5: Educational background and Emiratis' choices

\begin{tabular}{|c|c|c|c|c|c|c|}
\hline \multirow[b]{2}{*}{ Options } & \multicolumn{2}{|c|}{ "Yes" } & \multicolumn{2}{|c|}{ "No" } & \multicolumn{2}{|c|}{ "It depends" } \\
\hline & $N$ & $\%$ & $N$ & $\%$ & $N$ & $\%$ \\
\hline
\end{tabular}

Case 1: A patient is almost certain to die within the next six months. Should the doctor tell the patient?

$\begin{array}{lllrlrr}\text { Uneducated }(\mathrm{N}=13) & 4 & 31 \% & 9 & 69 \% & 0 & 0 \% \\ \text { Primary }(\mathrm{N}=22) & 6 & 27 \% & 11 & 50 \% & 5 & 23 \% \\ \text { Secondary }(\mathrm{N}=37) & 6 & 16 \% & 13 & 35 \% & 18 & 49 \%\end{array}$

$\begin{array}{lrrrrrr}\text { Secondary }(\mathrm{N}=37) & 6 & 16 \% & 13 & 35 \% & 18 & 49 \% \\ \text { Higher education }(\mathrm{N}=28) & 11 & 39 \% & 9 & 32 \% & 8 & 29 \%\end{array}$

Case 2: A patient has a $50 \%$ chance of dying within the next six months. Should the doctor tell the patient?

$\begin{array}{llllllr}\text { Uneducated }(\mathrm{N}=13) & 6 & 46 \% & 7 & 54 \% & 0 & 0 \% \\ \text { Primary }(\mathrm{N}=22) & 9 & 41 \% & 11 & 50 \% & 2 & 9 \% \\ \text { Secondary }(\mathrm{N}=37) & 9 & 24 \% & 14 & 38 \% & 14 & 38 \% \\ \text { Higher education }(\mathrm{N}=28) & 6 & 22 \% & 11 & 39 \% & 11 & 39 \%\end{array}$

Case $2\left(\mathrm{chi}^{2}=1.69, \mathrm{df}=2, \mathrm{p}>0.05\right)$. The responses of different education groupings are shown in table 5 .

When asked how much they believed of what doctors in the UAE told them, only $11 \%$ of the Emiratis interviewed replied "everything", $28 \%$ selected "most", $20 \%$ "half", $36 \%$ "some" and 6\% "nothing".

Twenty-eight per cent of Emiratis reported that someone close to them had died during the previous six months as a result of an accident or a condition which had not been identified beforehand as terminal. Their responses did not differ significantly from people without this experience when judging Case $1\left(\mathrm{chi}^{2}=2.90, \mathrm{df}=2, \mathrm{p}>0.05\right)$, Case $2\left(\mathrm{chi}^{2}=\right.$ $2.60, \mathrm{df}=2, \mathrm{p}>0.05)$ or when asked whether they would want the doctor to tell them if they had only a short time to live $\left(\mathrm{chi}^{2}=3.29, \mathrm{df}=2, \mathrm{p}>0.05\right)$.

Asked how many times they had a managed a patient similar to Case 1 (Case 2) during the past two years, 32\% (36\%) of doctors said "never", $34 \%$ $(26 \%)$ recalled fewer than five cases, $10 \%(24 \%) 5-$ 9 cases, and $24 \%(14 \%)$ ten or more cases. Asked whether or not Emirati patients resembled patients in the West in terms of wishing to be informed when their prognosis was poor, $76 \%$ of the doctors interviewed stated that they were different.

\section{Discussion}

LIMITATIONS OF THE STUDY

The current study investigated the opinions of convenience samples of 100 Emiratis and 50 doctors. Before the current findings can be assumed to be robust, they must be replicated with larger groups of subjects. The samples appear reasonably representative with regard to the specialties of doctors ${ }^{8}$ and the demographic features of the Emirati population. ${ }^{9}$ Due to the expansion of government-sponsored health services and a sustained high birth-rate, the age profile of the Emirati population is pyramidal. Another consequence of development is that age and education covary: most educated people are young and most older people are uneducated. Future studies to explore the opinions of different subgroupings (for example, the old, the educated, the sick, those who have experienced treatment abroad) will require stratified sampling to control related factors.

\section{CONTRASTING DOCTORS' AND EMIRATIS' OPINIONS}

In the current study, the doctors emerged as significantly less in favour of informing terminally ill patients than were the Emiratis. The disparity was evident whether the patient was described as almost certain to die during the next six months or as having a $50 \%$ chance of surviving, and even when it was made clear that a patient wished to be told. Emiratis who had personal experience of such situations did not make significantly different choices, and twothirds of the doctors interviewed claimed to have managed comparable patients during the past two years. These findings suggest that there may be many terminally ill patients in the UAE who would wish to be informed of this by their doctor who are never informed. Further studies, exploring specific areas of clinical practice, are needed to substantiate whether, and under what circumstances, this problem arises.

It is important to recognise that the modal response by Emiratis was "No, the doctor should not tell the terminally ill patient". Do doctors' responses, therefore, signify a sensitivity to local preferences? The answer is "No". Given the lack of consensus among Emiratis, a more empathic response would have been "It depends".

\section{DOES THE PATIENT ASK FOR INFORMATION?}

Both Emiratis and doctors were sensitive to whether or not the patient was specifically identified as requesting information. In the case of someone almost certain to die during the next six months, stipulating that the patient was asking for information raised the proportion of Emiratis who felt that the doctor should tell from a quarter to nearly $60 \%$, and reduced those selecting "It depends" from almost a third to $3 \%$. The effect on doctors was to raise the percentage who judged the patient should be told from $8 \%$ to $42 \%$, while reducing those selecting "It depends" from over half to less than a quarter. Uncertainty about whether the patient would or would not wish to be told is, therefore, a major factor causing respondents to choose "It depends". Nonetheless, about a third of respondents (both doctors and Emiratis) consistently stated that patients should not be told; making it clear that the patient wishing to be informed did not alter their stand.

\section{SURVIVAL ODDS}

Emiratis were not sensitive to changing survival odds. It may be that they regarded both patients (Case 1 and Case 2) as substantially at risk of dying or that they held unequivocal views about informing. Yet another possibility is that non-medically qualified respondents had difficulty appreciating the risk 
differential selected, ${ }^{10}$ but this was not suspected by interviewers. Changing survival odds, on the other hand, did affect doctors' choices. When it was uncertain whether the patient would wish to be informed, reducing the risk that the patient would die almost doubled those stating that the patient should not be informed (from $38 \%$ to $70 \%$ ). Even when the patient was identified as asking for information, increasing survival caused a one-third drop in the number of doctors who stated that the patient should be informed.

It is important to question whether changing survival odds justifies any change in behaviour from doctors. Case 2 did not imply that death was no longer a consideration; and information from other cultures suggests that some people benefit from having time to prepare for their death. It is also known that patients differ greatly in how they view statistical odds - a major risk for one person is considered a remote possibility by another. ${ }^{10}$ Viewed from this perspective, it is risky for the doctor to presume how patients will evaluate their prognosis or what choices and adjustments they will opt to make. Doctors cannot safeguard beneficence without input from the patient.

Many factors could create the disparity observed between patients' wishes for information and doctors' willingness to inform. Some doctors may be giving priority to other considerations, some may genuinely misunderstand what patients expect, others may be unaware of the potential benefits of informing or doubtful of their ability to handle the task and deal with the reactions of patients and families. There may even be some doctors who will resist informing until forced to do so by patient pressure, professional codes or legislation.

\section{Ethical principles}

Ethically, the group of doctors whose responses are most worrying are those who indicated that they would override the patient's expressed wish to be informed. About a third of the doctors interviewed in the UAE endorsed this approach. Given the fact that doctors had available to them the "It depends" category, such a reaction appears to reflect an entrenched opposition to informing rather than a recognition that withholding information is sometimes justified. The fact that a third of Emiratis thought the same does not justify these doctors' responses. Doctors are expected to be better versed in ethical principles than lay people, so that they can safeguard ethical standards and serve as mentors for their patients. The fact that patients did not allude to ethical principles is not surprising ${ }^{5}$ but the fact that the doctors interviewed did not do so is worrying. Most of the doctors and Emiratis interviewed considered that there were circumstances in which doctors should inform the family but not the terminally ill patient. These were not limited to cases of mental incompetence, psychological instability $\overrightarrow{\overrightarrow{\mathrm{Ar}}}$ immaturity. None of the doctors spoke of any ethiọal dilemma in conjunction with informing only family, even though such an action would be con sidered contentious in many countries. ${ }^{1}$ Almost 90 of the Emiratis interviewed claimed not to believe क्ष्र they were told by doctors. It would be worth explo ing to what extent this lack of trust is linked $P$ doctors' unwillingness to provide information. के

\section{ETHICAL NORMS}

In terms of cultural considerations, the data can $\overrightarrow{\mathrm{be}}$ looked at in two ways: one is to contrast the choices of Emiratis with those of people elsewhere, the second is to look for consensus among UAE respoin dents.

Faced with the case of a patient who was almost certain to die, Greek respondents were equally sphit between the "Yes", "No" and "It depends" options. ${ }^{5}$ Emiratis, on the other hand, exhibited $\vec{a}$ 27:42:31 split. Previous studies suggest that had the current study been carried out in Western Europe North America, many more lay people and docto $\overrightarrow{a s}$ would have endorsed informing. Looked at in this way, countries do differ in their choice profiless Indeed, the majority of doctors interviewed claime that Western patients and Emirati patients differ their wishes about being informed when their prog nosis is poor.

The current data, however, revealed no consens녕 among Emiratis as to whether or not doctors shouki inform terminally ill patients. Knowing that the patient is a UAE national, therefore, does net provide a sound basis for predicting what choicés he/she will make. A similar picture was found Greece. ${ }^{5}$ Before an attitude or opinion can qualify af a norm, it must be characteristic of a society or sub. culture. In terms of the cases described, there was no evidence of a normative opinion among Emiratis to whether or not doctors should inform terminalby ill patients.

As was also found in the much larger Gre study, ${ }^{5}$ men and women did not differ in the response profiles. People's choices were not affecte\& by having experienced comparable situations. In the Greek study, the experience of losing a parent wâs associated with a greater frequency of " $\mathrm{N} \omega^{\text {" }}$ responses. When asked whether terminally i patients should be informed, older/less-educate Emiratis replied "Yes" or "No", whereas a third \&f the younger/better educated respondents selected "It depends". This same pattern was evident in the Greek study. ${ }^{5}$ Further investigations are indicated separate the effects of age and education and esta lish the reasons for their impact. Younger/better educated people's greater experience of travelling abroad and their greater exposure to Western med: may play a role. They never, however, invoket ethical considerations or patients' rights wh explaining their choices. This suggests that if 
exposure to Western medical practice is having any impact, it is at the level of seeing how doctors elsewhere behave without fully appreciating why. Some of the doctors interviewed, albeit not many, unreservedly endorsed informing patients. They represent a potentially very powerful influence on their patients and their patients' families.

\section{PERSONAL CHOICES}

Despite the lack of consensus among Emiratis about how doctors should deal with a patient who is soon to die, they were seldom ambiguous about how they would want to be handled themselves: only $4 \%$ answered "It depends". Individuals' personal wishes often deviated from their statements about how doctors should in principle react. Of the Emiratis who said that they would want to be told if they had only a short time to live, only a third had stated that the doctor should tell the patient described in Case 1, a third had answered "No" and a third "It depends". What these people thought was right for others proved no guide to what they wished for themselves. The disparity was not caused by respondents selecting "It depends" when they were uncertain about other people's circumstances. Many who unambiguously selected "Yes" for themselves chose "No" when judging Case 1 .

\section{Conclusions}

The salutary conclusion is that in the UAE, and probably elsewhere, opinion profiles do not offer a useful guide to what information a particular patient will want; both because of heterogeneity and because in principle judgments often do not equate with personal wishes. This is not to suggest that it is worthless to explore cultural factors. Doctors need to be aware of potentially relevant cultural issues and conversant with culturally acceptable ways of exploring patients' needs and providing them with information. Looking broadly at the issues of whether, when and how to inform, Surbone commented: "I believe Italians should not borrow the American way, but that they should learn from Americans and try to find a better Italian way". ${ }^{6}$ It has to be recognised, however, that one way will not optimise individual care. Even in the most homogeneous societies, patients do differ and doctors should avoid adopting stereotyped responses. Guidelines about how to translate ethics into medical practice were never intended to become autonomous requirements. For each patient, the doctor should consciously decide whether or not to inform after weighing the implications of both. For doctors practising in multicultural settings, or in a society where relevant opinions are diverse or changing, exploring patients' wishes in an empathic way constitutes a major challenge.

Ann Harrison PhD, BSc(Econ), AFBPsS, CPsychol, is Associate Professor of Medical Psychology in the Department of Behavioural Sciences, St George's University School of Medicine, Grenada. Ahmed M H Al-Saadi, Ali S O Al-Kaabi, Mohammed R S Al-Kaabi, Saif $S M$ Al-Bedwawi, Saif $O M$ Al-Kaabi and Salem $B S A l-N e a i m i$ are fourth-year medical students with the United Arab Emirates University Faculty of Medicine and Health Sciences; they completed the study in partial fulfilment of the Human Sciences Module of the MBBS Degree.

\section{References}

1 Pellegrino ED. Is truth telling to the patient a cultural artifact?[editorial]. Fournal of the American Medical Association 1992; 268: 1734-5.

2 Newall DJ, Gadd EM, Priestman TJ. Presentation of information to cancer patients: a comparison of two centres in the UK and USA. British fournal of Medical Psychology 1987; 60: 127-131.

3 Thomsen OO, Wulff HR, Martin A, Singer PA. What do gastroenterologists in Europe tell cancer patients? Lancet 1993; 341: 473-6.

4 Interdisciplinary Group for Cancer Care Evaluation. What doctors tell patients with breast cancer about diagnosis and treatment: findings from a study in a general hospital. British fournal of Cancer 1986; 54: 319-326.

5 Dalla-Vorgia P, Katsouyanni K, Garanis TN, Touloumi G, Drogari P, Koutselinis A. Attitudes of a mediterranean population to the truth-telling issue. Journal of Medical Ethics 1992; 18: 67-74.

6 Surbone A. Truth telling to the patient [letter]. Fournal of the American Medcial Association 1992; 268: 1661-2.

7 Liberati A, Meyerowitz B. Truth telling: a cultural or individual choice?[letter]. Fournal of the American Medcial Association 1993; 269: 989.

8 Ministry of Planning, United Arab Emirates. 1990 Annual Statistical Book.

9 Preventative Medicine Department, Ministry of Health, United Arab Emirates. 1990 Annual Report.

10 McNeil BJ, Pauker SG, Sox HC, Tversky A. On the elicitation of preferences for alternative therapies. New England fournal of Medicine 1982; 306: 1259-1262. 